\section{The effects of aversive levels of white noise on consummatory behavior}

\author{
W. RASBURY and K. SHEMBERG \\ Bowling Green State University, Bowling Green, Ohio 43402
}

The present study was designed to investigate the effects of aversive levels of white noise on consummatory behavior. Three levels of sound were chosen for study $(90,100$, and $110 \mathrm{~dB})$. The results showed that $\mathrm{Ss}$ in the sound treatment conditions consumed significantly greater quantities of food on the second sound treatment day than did Ss in the no-sound control condition. In addition, there were no systematic differences in consummatory behavior among the three treatment conditions.

The available literature indicates that the effectiveness of auditory stimulation as a noxious stimulus varies as a function of the experimental conditions employed (Bolles \& Seelbach, 1964). The present study focused upon the effects of aversive levels of white noise on eating behavior and its possible application as an aversive stimulus in situations which involve an appetitive response. It is difficult to predict the specific effects of the sound levels presently utilized upon eating. Some previous work on the effects of aversive electric shock on eating behavior has indicated that lower shock intensities facilitate the eating response, while high levels of shock stimulation produce inhibition (Bolles, 1967). A similar relationship may emerge relative to white noise stress and eating behavior. However, no claim is made here as to the direct aversive equivalence of the sound levels presently chosen and the shock intensities previously utilized. The specifjc purpose of the present study was to provide some information regarding possible facilitative and/or inhibitory relationships between white noise as an aversive stimulus and the rats' eating behavior. Such information may be useful in future research on aversive stimulation and the consummatory response.

Three levels of white noise which have been shown to be aversive were chosen for study (Campbell \& Bloom, 1965; Halpren \& Lyon, 1966). Ss in each of the sound conditions $(90$, 100 , and $110 \mathrm{~dB}$ white noise) received one $20-\mathrm{min}$ feeding session per day for 5 days, with sound administered on Days 2 and 4 . A no-sound control group was used to provide an index of consummatory behavior that was independent of the sound presentations.

\section{SUBJECTS}

The Ss were 60 Holtzman male rats, 90 days old and weighing $280-290 \mathrm{~g}$; they were assigned randomly to the four conditions $(N=15$ per condition: $90-, 100-$, and 110-dB white noise, and no-sound control).

The Ss were reduced to $90 \%$ of thei ad lib feeding weight over a 5-day during each $20 \cdot \mathrm{min}$ test period. period by feeding them $5 \cdot 8 \mathrm{~g}$ of Purina Lab Chow per day. Water was always provided ad lib in the home cage but withheld during test sessions. Daily feeding in the home cage occurred at a time corresponding to the S's subsequent feeding in the test apparatus. Following the initial 5-day reduced feeding schedule, each $\mathrm{S}$ was placed in the test chamber for a 10-min eating acclimation session. One 45-mg Noyes pellet was dispensed into the feeding cup. As soon as $\mathrm{S}$ removed the pellet another was dispensed, and so on. This feeding procedure was used in all subsequent test sessions.

The day following eating acclimation, Ss received the first of five daily $21 \cdot \mathrm{min}$ feeding sessions. The first minute of each session was a warm-up period, and pellets consumed then were not counted as part of the test session. The number of pellets consumed during each minute of the remaining $20 \mathrm{~min}$ was recorded. For $\mathrm{Ss}$ in the three sound conditions, Days 1, 3 , and 5 were used as base rate measures of food consumption. Sound was presented on Days 2 and 4 and began at the end of the warm-up period and lasted throughout the session. Ss in the no-sound condition received five no-sound test sessions. Ss were weighed immediately before and after each test session.

\section{RESULTS AND DISCUSSION}

A separate single-factor analysis of variance was performed on the food-consumption data for each of the five test sessions. There were no differences in eating behavior among the four groups on Days $1,2,3$, or 5 . However, on Day 4 , the second sound treatment day, a significant facilitation effect emerged $(\vec{F}=4.69, \mathrm{df}=3 / 56$, $\mathrm{p}<.01)$. On this day, $\mathrm{Ss}$ in the

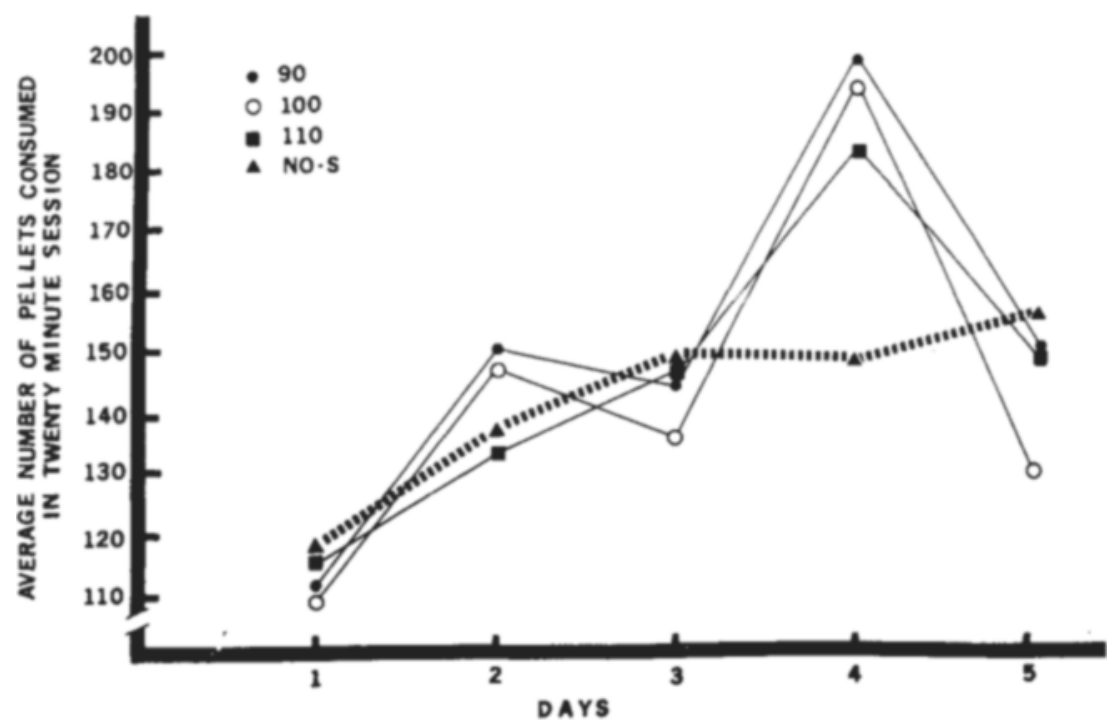

Fig. 1. The average number of food pellets consumed by Ss in each condition 
treatment conditions ate significantly more than did control Ss (Newman-Keuls, $p<.05$ ). There were no differences among the treatment groups in food consumption (see Fig. 1).

Separate single-factor analyses were performed on each successive $5 \mathrm{~min}$ of each test session. These analyses were conducted to determine if there were any specific within-session patterns of consummatory behavior that differed from those displayed in Fig. 1 . Specific within-session differences did occur during the first $5 \mathrm{~min}$ of the first treatment session. Ss in the 100-and $110-\mathrm{dB}$ conditions exhibited significant reductions in food consumption relative to that of $\mathrm{Ss}$ in the $90-\mathrm{dB}$ and no-sound control conditions $(\mathrm{F}=7.07, \mathrm{df}=3 / 56$, $\mathbf{p}<01$; Newman-Keuls comparisons, $p<.05$ ). No other within-session differences appeared.

Analyses performed on the presession warm-up data did not reveal any systematic differences in the number of food pellets consumed by Ss in the four conditions. In addition, there were no systematic differences in presession body weight among the conditions.

There were no significant differences in total session eating behavior among $S s$ in the three white-noise conditions utilized. The most striking finding of the present research was that no substantial inhibition of eating occurred. In contrast to inhibition, there was a marked facilitative effect on the second sound treatment day. This facilitation is similar to effects previously reported, when mild levels of electric shock were presented to Ss in an appetitive situation (e.g., Sterritt \& Shemberg, 1963; Sterritt, 1965).

It is possible that failure to produce inhibition was a function of the relatively long testing sessions and that the most aversive aspects of sound underwent some adaptation. The fact that an initial period of inhibition occurred in the 100- and 110-dB conditions, which gave way to facilitation, provides some support for this possibility. It is possible to suggest that the facilitative effect is in part an artifact produced by the delivery of white noise from above, which forced animals' heads down to the feeding cup area. Observation of the Ss strongly indicates that this is not the case. The onset of white noise typically produced initial behaviors which were incompatible with eating (e.g., face washing), which were then followed by a rapid approach to the foodcup.

In summary, the current data suggest that the presentation of substantial durations of white noise as an aversive stimulus in an appetitive

situation is of little value where the primary concern is with measuring disruptive effects. Inhibitory effects are small and of short duration. The present project provides no substantive information regarding possible processes underlying the observed facilitative effect, and this finding itself appears to be worthy of future exploration.

\title{
REFERENCES
}

BOLLES, R. Theory of motivation. New York: Harper \& Row, 1967.

BOLLES, R., \& SEELBACH, S. Punishing and reinforcing effects of noise onset and termination for different responses. Journal of Comparative \& Physiological

\section{Effects of strain differences and D-amphetamine sulfate on avoidance performance*}

\author{
BARBARA J. POWELL \\ Malcolm Bliss Mental Health Center and Washington University \\ St. Louis, Mo. 63104 \\ and \\ DOTTY J. HOPPER \\ University of Missouri, St. Louis, Mo. 63121
}

The purpose of this experiment was to investigate strain differences and amphetamine effects on avoidance performance of the Maudsley MR and MNR strains of rats. It was predicted that with amphetamine avoidance performance of the nonreactive strain would be enhanced more than that of the reactive strain. There was no significant difference in performance between the two strains; however, groups which received amphetamine had better performance than those receiving placebo.

Strain differences are known to influence avoidance performance of rats (Zerbolio, Reynierse, \& Denny, 1965; Wilcock, 1968). D-amphetamine sulfate, a stimulant drug, has been shown to have a facilitating effect on avoidance performance (Hearst \& Whalen, 1963; Kulkarni \& Job, 1967). In a strain by drug avoidance learning study, Powell, Martin, \& Kamano (1967) reported differential effects of amobarbital sodium on performance of the Tryon strains. Avoidance performance of the $\mathrm{S} 1$ strain was facilitated more by $20 \mathrm{mg} / \mathrm{kg}$ and debilitated less by $40 \mathrm{mg} / \mathrm{kg}$ than was that of the S3 strain. Open field testing indicated that these strains also differed in emotionality, with the S1s found to be more emotional than the S3s; thus, it was inferred that the drug effects interacted with emotionality. Since amobarbital normally has a depressant effect on activity, with d-amphetamine the opposite results might be expected, e.g., amphetamine should improve performance of relatively nonemotional rats and should hinder performance of more emotional rats. Such a hypothesis is in

* Supported in part by USPHS Research Grant MH-09247. keeping with the inverted U-shaped function.

This study investigated the effects of d-amphetamine sulfate on the avoidance performance of the Maudsley reactive (MR) and nonreactive (MNR) strains of rats, which are said to differ in emotionality (Broadhurst, 1969).

$$
\text { SUBJECTS }
$$

The Ss were 24 female Maudsley rats, 12 from the MNR and 12 from the MR strain. Each strain was divided into equal drug and placebo groups. Ss were 80.120 days old, were maintained on food and water ad lib, and were housed individually throughout testing.

\section{APPARATUS}

The apparatus was a shuttlebox, $24 \times 3 \frac{1 / 2}{2} \times 5$ in., constructed of clear Plexiglas with a grid floor. The top of the box was hinged for inserting and removing the animals. The box was divided into two sections by a black cloth curtain. A Hunter timer was used for timing response latency and the CS-US interval. An Eico audiogenerator produced the CS $($ tone $=400$ cycles $/ \mathrm{sec})$. Testing took place in a dimly lighted room.

$$
\text { PROCEDURE }
$$

Each S was subjected to 20 trials of 